\title{
NELS JOHANN LENNES
}

Nels Johann Lennes was born June 15, 1874, in Henne, Norway. He came to the United States in 1890, unable to speak the English language. He entered the University of Chicago in 1896 and received from that institution a bachelor's degree in 1898, a master's degree in 1903, and a Ph.D. degree in 1907, having been influenced by E. H. Moore and others at Chicago. He was married and the father of three children.

From 1898 to 1907 he taught in Chicago high schools in addition to doing graduate study at the University. He was an instructor at Massachusetts Institute of Technology from 1907 to 1910 and at Columbia University from 1910 to 1913 . He became Professor of Mathematics and Chairman of the Department at Montana State University in 1913 and held that position until his retirement in 1944. His death occurred at Missoula, Montana, November 21, 1951.

He was a mathematician of excellent talent, one of the precursors of modern abstract mathematics, who made a number of interesting contributions, and who in different circumstances would very likely have made many more. In the latter part of his life he wrote nearly one hundred textbooks which were widely used. The bibliography below contains only his scientific work.

His interests were mainly in functions of a real variable, foundations of geometry, and the topology of euclidean spaces. He proved [9] that a polyhedron separates space. In the same paper he also proved that a polygon can be divided into triangles and that the corresponding theorem is true for polyhedra. Apparently none of these facts had been proved earlier. Here he was motivated by definitions of area and volume. He also gave self-dual axioms for projective geometry. In one note [3] he analyzed conditions under which the Heine-Borel theorem is valid and pointed out that if points at infinity are added the theorem is not restricted to bounded sets. Systematic use of the "Heine-Borel property" (i.e. compactness) was a feature of the book [4] on real function theory.

In the paper [10] which is one of his best the term connected set for subsets of the plane is defined as follows: "A set of points is a connected set if at least one of any two complementary subsets contains a limit point of points in the other set." In [8] the same definition was given for subsets of any euclidean space. For closed sets a similar definition had been given by Jordan, Cours d'analyse, vol. I, 1893 , p. 25 . Using this definition Lennes characterized an arc as a 
compact connected set containing two distinct points $a$ and $b$ and such that no proper connected subset contains $a$ and $b$. This characterization which was given for the plane and later euclidean spaces extends unchanged to more general spaces, and remains in wide use.

\section{BiBLIOGRAPHY}

1. Remarks on a proof that a continuous function is uniformly continuous, Ann. of Math. (2) vol. 6 (1905) pp. 86-88.

2. Volumes and areas, Trans. Amer. Math. Soc. vol. 6 (1905) pp. 486-490.

3. Note on the Heine-Borel Theorem, Bull. Amer. Math. Soc. vol. 12 (1905-06) pp. 395-398. Hafner.

4. Infinitesimal analysis (with O. Veblen), Wiley, 1907, pp. 1-230; reprinted by

5. Note on the variation of the definite integral, Bull. Amer. Math. Soc. (2) vol. 13 (1906-07) pp. 10-20. 22.

6. Concerning the improper definite integral, Amer. J. Math. vol. 29 (1906) pp. 13-

7. The motion of a ball on a billiard table, Amer. Math. Monthly vol. 12 (1905) pp. $152-155$.

8. Abstracts, Bull. Amer. Math. Soc. vol. 17 (1910-1911) pp. 525-526.

9. Theorems on the simple finite polygon and polyhedron, Amer. J. Math. vol. 33 (1911) pp. 37-62. 326.

10. Curves in non-metrical analysis situs, Amer. J. Math. vol. 33 (1911) pp. 287-

11. The fundamental theorem of the differential calculus, Bull. Amer. Math. Soc. vol. 23 (1916-17) pp. 488-490.

12. A direct proof of the theorem that the number of terms of the expansion of an infinite determinant is of the same potency as the continuum, Bull. Amer. Math. Soc. vol. 18 (1911-12) pp. 22-24.

13. Review of "Theory of sets of points" (W. H. Young and Grace Chisholm Young), Bull. Amer. Math. Soc. vol. 18 (1911-12) pp. 24-30.

14. Duality in projective geometry, Ann. of Math. (2) vol. 13 (1912) pp. 11-16.

15. A set of independent postulates for projective geometry, Amer. Math. Monthly vol. 18 (1911) pp. 174-182.

16. A necessary and sufficient condition for the uniform convergence of a certain class of infinite series, Bull. Amer. Math. Soc. vol. 18 (1911-12) pp. 66-70.

17. Concerning Van Vleck's non-measurable set, Trans. Amer. Math. Soc. vol. 14 (1913) pp. 109-112.

18. A brief study in non-mathematical logic, The Mathematics Teacher vol. 16 (1923) pp. 242-246.

Deane Montgomery and Oswald Veblen 